\title{
Miniaturized Photoacoustic Trace Gas Sensing using a Raman Fiber Amplifier
}

\author{
Ralf Bauer, Thomas Legg, David Mitchell, Gordon M. H. Flockhart, George Stewart, Walter \\ Johnstone, and Michael Lengden
}

\begin{abstract}
This paper presents the development of a Raman fiber amplifier optical source with a maximum output power of $1.1 \mathrm{~W}$ centered around $1651 \mathrm{~nm}$, and its application in miniaturized 3D printed photoacoustic spectroscopy (PAS) trace gas sensing of methane. The Raman amplifier has been constructed using $4.5 \mathrm{~km}$ of dispersion shifted fiber, a $1651 \mathrm{~nm}$ DFB seed laser and a commercial 4W EDFA pump. The suppression of stimulated Brillouin scattering (SBS) using a high frequency modulation of the seed laser is investigated for a range of frequencies, leading to an increase in optical output power of the amplifier and reduction of its noise content. The amplifier output was used as the source for a miniature PAS sensor by applying a second modulation to the seed laser at the resonant frequency of $15.2 \mathrm{kHz}$ of the miniature 3D printed gas cell. For the targeted methane absorption line at $6057 \mathrm{~cm}^{-1}$ the sensor system performance and influence of the SBS suppression is characterized, leading to a detection limit (1б) of $17 \mathrm{ppb}$ methane for a signal acquisition time of $130 \mathrm{~s}$, with a normalized noise equivalent absorption coefficient of $4.1 \cdot 10^{-9} \mathrm{~cm}^{-1} \mathrm{~W} \mathrm{~Hz}^{-1 / 2}$ for the system.
\end{abstract}

Index Terms-Miniaturized fiber optic sensor, 3D printed photoacoustic trace gas sensor, Raman fiber amplifier system, SBS suppression

\section{INTRODUCTION}

$\mathrm{L}$ ASER excited photoacoustic spectroscopy (PAS) has been used as a tool for trace gas detection with high sensitivity and gas species selectivity in a large number of application fields ranging from medical diagnostics [1], industrial process control [2] and gas leak detection [3] to environmental

Manuscript received February 16, 2015. This work was supported in part by the EPSRC under Grant EP/K034758/1.

R. Bauer, G. Flockhart, G. Stewart, W. Johnstone, and M. Lengden are with the Centre for Microsystems and Photonics, Department of Electronic and Electrical Engineering, University of Strathclyde, Glasgow, G1 1XW, U.K. (e-mail: ralf.bauer@strath.ac.uk; gordon.flockhart@strath.ac.uk; g.stewart@strath.ac.uk; w.johnstone@ strath.ac.uk; michael.lengden@ strath. ac.uk).

T. Legg was with the Centre for Microsystems and Photonics, Department of Electronic and Electrical Engineering, University of Strathclyde, Glasgow, G1 1XW, U.K, and is now with Gooch \& Housego, Torquay, TQ2 7QL, U.K. (e-mail: tlegg@goochandhousego.com).

D. Mitchell was with the Centre for Microsystems and Photonics, Department of Electronic and Electrical Engineering, University of Strathclyde, Glasgow, G1 1XW, U.K, and is now with Coherent Scotland Ltd, Glasgow, G20 0XA, U.K. (e-mail: david.mitchell@ coherent.com).

Copyright (c) 2015 IEEE. Personal use of this material is permitted. However, permission to use this material for any other purposes must be obtained from the IEEE by sending a request to pubs-permissions@ieee.org. monitoring [4]. Based on Bell's work in 1880 demonstrating the generation of an acoustic response to modulated light absorption in a medium [5], a multitude of systems have been demonstrated since the availability of narrow linewidth lasers as excitation sources, with record detection limits in the partsper-trillion range [6]. PAS provides a background free optical spectroscopy system, with an inherent potential for miniaturization due to acoustic resonance amplitudes increasing for small scale devices. Miniaturized PAS systems have been demonstrated by scaling conventional resonant gas cell designs, employing wafer bonding [7], small scale manufacturing [3] or 3D-printing [8]. A further approach is by using resonant acoustic detectors in the form of cantilevers [9] or quartz tuning forks [10]. The combination of resonant detectors and an additional acoustic resonator can also be used [11], with the two resonances needing to be matched. In order to expand the detection limits with a single gas cell, two general routes are possible: a commonly used move to midinfrared excitation, where a higher gas absorbance is present, and/or power scaling of the source laser.

Power scaling of near-infrared laser diodes at telecommunication wavelengths is well developed with fiber based systems, notably using an erbium doped fiber amplifier (EDFA) operating at $1.54 \mu \mathrm{m}$ [12] and an ytterbium doped fiber amplifier (YDFA) operating at $1.06 \mu \mathrm{m}$ [13]. Amplification using an EDFA has been applied in PAS for detection of gas species with finger print regions in the amplifier wavelength band of $1530 \mathrm{~nm}$ to $1565 \mathrm{~nm}$ [14], [15]. However, fiber based amplifier systems outside this wavelength range have, to the best of our knowledge, not been demonstrated so far for trace gas sensing. To access spectroscopic finger print regions of trace gases outside of these wavelength bands further wavelength coverage of optical fiber amplifiers is needed. One way to achieve this is to utilize stimulated Raman scattering (SRS) inside silica fibers, which have a Raman gain bandwidth of up to $40 \mathrm{THz}$ with a broad peak at a frequency shift of around $13 \mathrm{THz}$ [16]. This can lead to a narrowband tunable amplified signal at a targeted wavelength given the correct pump and seed laser wavelength combination. Fiber based amplifiers utilizing SRS were first shown in the late 1960s [17], with systems for telecommunication applications only starting to appear in the late 1990s. Current applications of SRS amplifier systems include the use for laser guide stars in astronomy [18], and the use in telecommunications systems [19]. In all cases, the 
required high pump and desired Raman output powers inside the fiber lead to further unwanted nonlinear scattering effects like stimulated Brillouin scattering (SBS). This backwards propagating acoustic phonon scattering effect has a bandwidth of approximately $10-50 \mathrm{MHz}$ with an associated frequency shift equal to $\sim 10 \mathrm{GHz}$ [16]. This phenomena also limits the achievable Raman amplified output power through the introduction of a strong additional loss to both the pump and Raman amplification. The suppression of SBS is therefore necessary for high power systems to improve both the overall Raman output power and to avoid optical noise generated in sidebands of the Raman output. This suppression has been investigated in the form of a variation of the fiber core size [20], an arbitrary strain distribution over the fiber length [21] or a modulation of the seed laser to broaden its linewidth [22].

In this paper, we present the novel application of a custom Raman fiber amplifier as the source for a fiber coupled miniaturized 3D printed PAS trace gas sensor. The Raman fiber amplifier is tailored for signals at $1651 \mathrm{~nm}$ for the interrogation of methane absorption lines and has an output power above $1 \mathrm{~W}$ and a frequency modulation scheme to suppress SBS. This technique can be applied with a variety of seed laser and pump combinations for detection of a vast number of further trace gases (e.g. $\mathrm{H}_{2} \mathrm{O}_{2}, \mathrm{CO}, \mathrm{CO}_{2}, \mathrm{~N}_{2} \mathrm{O}$ ). In Section II the theoretical background for the design of the 3D printed PAS sensor will be presented, followed by the details of the gas cell design and fabrication of the 3D printed sensor in Section III. Section IV will detail a theoretical optimization of the Raman amplifier fiber length and the associated simulation of the final system. Section V will then detail the experimental characterization of the Raman amplifier system, investigating the dependency of the output power on the SBS suppression via high frequency modulation. Section VI will conclude with the experimental characterization of the Raman amplifier combined with the 3D printed sensor for methane trace gas sensing in the parts-per-billion (ppb) range.

\section{Photoacoustic SPECTROSCOPY}

A sensor signal is generated in PAS through the absorption of light by a target gas species, in general by using a narrow linewidth laser modulated at an acoustic frequency. The associated periodic heating and cooling of the target gas leads to the generation of an acoustic wave, which is sensed by a microphone or equivalent pressure sensor. To increase the strength of the acoustic wave, and thereby the sensitivity of the sensor, acoustic resonators are in general employed. These can be in the form of a gas cell with a resonant behavior, a resonant sensor or a combination of both.

Modulated laser-gas interactions are typically achieved through either the modulation of the laser drive current, resulting in both wavelength and intensity modulation of the laser output, or through the direct intensity modulation of the laser output via an optical chopper or electo-optical modulator. In this work we use the methods of conventional wavelength modulation spectroscopy (WMS) [23]-[30] where modulation is applied to the laser drive current and the first or second harmonic of the modulation frequency is detected at the output by a lock-in amplifier and related to the gas parameters. The same modulation technique has been widely applied in PAS [31]-[34] with the advantage that the first harmonic signal from the microphone has a zero or very low background level. For detection of the first harmonic at the resonant frequency $f_{0}$ of a resonant gas cell operating at a longitudinal resonance, the acoustic pressure change $p\left(\omega_{0}\right)$ can be approximated to [35], [36]:

$$
p\left(\omega_{0}, t\right) \approx \frac{\alpha_{0} C l(k-1) Q}{\omega_{0} V}\left\{\begin{array}{l}
a_{1} P_{L} \cos \left(\omega_{0} t-\psi\right) \\
+a_{0} \Delta P_{L} \cos \left(\omega_{0} t\right) \\
+\frac{1}{2} a_{2} \Delta P_{L} \cos \left(\omega_{0} t-2 \psi\right)
\end{array}\right\}
$$

where $\omega_{0}$ is the angular resonance frequency, $\alpha_{0}$ is the absorption coefficient at the line centre of the gas sample, $C$ is the gas concentration, $l$ is the resonator length, $k$ is the specific heat ratio of the gas, $Q$ is the Q-factor of the resonator, $V$ is the volume of the resonator, $P_{L}$ is the laser power, $\Delta P_{L}$ is the intensity modulation, $a_{0}, a_{1}, a_{2}$ are the Fourier coefficients of the line-shape function and $\psi$ is the phase lag of the wavelength modulation. The significance of the various components in equation (1) is discussed in detail in [29] for conventional WMS and similar considerations apply to PAS.

Equation (1) indicates the direct proportionality of the primary component of the sensor signal with the laser power and therefore shows clearly the motivation behind using the Raman amplifier system developed and applied in this work. The miniaturization advantage of PAS gas sensors, targeted through the miniaturized 3D printed format in this work, can also be derived from (1) when the dimensional parameters influencing the Q-factor and the cell resonance frequency are included [36]. This leads to a pressure signal dependency of [8]:

$$
p\left(\omega_{0}\right) \propto \frac{1}{r} \sqrt{l}
$$

where $r$ is the radius of the resonator and $l$ its length. Equation (2) points to the ideal form of a long and thin longitudinal resonator achieving the highest possible acoustic pressure. A further dimensional constraint is imposed by the dependency of the resonance frequency on $l$, with a longer resonator length leading to lower resonance frequencies, resulting in a higher noise background.

Note also that the Fourier coefficients in equation (1) are dependent on the modulation index $m$, defined as [25], [26], [29]:

$$
m=\frac{\delta v}{\gamma}=\frac{\partial v}{\partial i} \frac{\delta i}{\gamma}
$$

where $\delta v$ is the laser frequency modulation amplitude, $\gamma$ is the half-width half-maximum of the gas absorption line and $\delta i$ is the laser drive current modulation amplitude employed to create the WM. The maximum output signal amplitudes are 
created for a modulation index $m=2.2$ for second harmonic demodulation and for $m=2$ for first harmonic demodulation for a Lorentzian line shape of the gas absorption line [25], [37]. In our previous work [8], we have shown that a first harmonic demodulation will result in higher sensor sensitivities for the 3D printed miniaturized gas sensor system. For the work discussed in this paper we will apply a dual modulation scheme to the laser drive current to create the WM associated with the acoustic resonance as well as a modulation to suppress SBS in the Raman amplifier.

\section{3D PRINTED GAS CELl DESIGN AND FABRICATION}

The miniature 3D printed gas cell is similar to the one described in our previous work [8] and is constructed from a photopolymerized acrylic resin (R11 from EnvisonTec). The longitudinal resonance of a cylindrical resonator with $10 \mathrm{~mm}$ length and $1.8 \mathrm{~mm}$ diameter (see Fig. 1) is used to amplify the generated PA signal, which is detected by a miniature microelectromechanical systems microphone (Wolfson Microelectronics WM7133). A more detailed description of the fabrication of the $25 \times 24 \times 15 \mathrm{~mm}^{3}$ cell with total internal volume of $350 \mathrm{~mm}^{3}$ can be found in [8].

An acoustic finite element model was built in COMSOL Multiphysics to simulate and confirm the operation frequency of the targeted first longitudinal resonance of the gas cell. The simulation model included the resonator, buffer volumes, gas inlet and outlet and microphone air cavities. It was built using a pressure acoustic model and the material parameters for a mixture of $1000 \mathrm{ppm} \mathrm{CH}_{4}$ in $\mathrm{N}_{2}$, given in the lower part of Table I. The resulting first longitudinal resonance frequency was simulated as $15.3 \mathrm{kHz}$, with its maximum acoustic pressure located at the microphone position.

\section{RAMAN AMPLIFIER AND SBS}

To generate the high optical powers desired for the generation of the PAS signals, a counter-propagating Raman fiber amplifier system has been designed. Amplification using SRS is based on the nonlinear scattering effect on optical phonons in the fiber. In silica fibers this nonlinear effect results in a

TABLE I

PARAMETERS FOR RAMAN AMPLIFIER AND GAS CELL SIMULATIONS

\begin{tabular}{lll}
\hline \hline \multicolumn{1}{c}{ Symbol } & \multicolumn{1}{c}{ Quantity } & \multicolumn{1}{c}{ Parameter value } \\
\hline$g_{r}$ & Raman gain coefficient & $4.4 \cdot 10^{-14} \mathrm{~m} / \mathrm{W}$ \\
$\varepsilon$ & Polarization factor & 0.55 \\
$g_{b, e f f}$ & Effective Brillouin gain coefficient & $4 \cdot 10^{-13} \mathrm{~m} / \mathrm{W}$ \\
$A_{e f f}$ & Mode area of fiber core & $5.2 \cdot 10^{-11} \mathrm{~m}^{2}$ \\
$\alpha_{P}$ & Fiber attenuation coefficient for the & $2 \cdot 10^{-4} \mathrm{~dB} / \mathrm{m}$ \\
& pump $(\lambda=1540 \mathrm{~nm})$ & \\
$\alpha_{S}$ & Fiber attenuation coefficient for the & $3 \cdot 10^{-4} \mathrm{~dB} / \mathrm{m}$ \\
& seed $(\lambda=1651 \mathrm{~nm})$ & \\
$P_{P}(L)$ & Pump input power & $4 \mathrm{~W}$ \\
$P_{R}(0)$ & Raman seed power & $6.5 \mathrm{~mW}$ \\
$P_{S B S}(L)$ & SBS seed power boundary condition & $1 \mu \mathrm{W}$ \\
\hline$\rho$ & Density of gas mixture & $1.12 \mathrm{~kg} / \mathrm{m}^{3}$ \\
$v$ & Speed of sound of gas mixture & $345 \mathrm{~m} / \mathrm{s}$ \\
$T$ & Temperature & $293 \mathrm{~K}$ \\
$p$ & Absolute pressure & $1 \mathrm{~atm}$ \\
$l$ & Resonator length & $10 \mathrm{~mm}$ \\
$d$ & Resonator diameter & $1.8 \mathrm{~mm}$ \\
\hline \hline
\end{tabular}

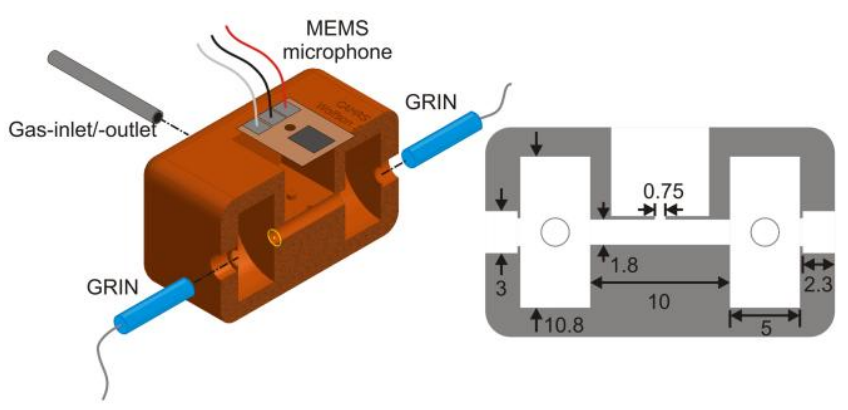

Fig. 1 Schematic half-cut of the 3D-printed PAS cell and cell cross-section including the cell dimensions (all in $\mathrm{mm}$ ).

Stokes frequency shift with a maximum Raman gain at a shift of $13.2 \mathrm{THz}$, leading to an optimum pump wavelength of $1531 \mathrm{~nm}$ for maximum amplification around $1651 \mathrm{~nm}$. This is conveniently located inside the output wavelength range of Cband EDFAs, which are commercially available with output powers in the Watt level range. The relatively low Raman gain coefficients in standard single mode fibers leads to long amplifier fiber lengths to achieve Raman output powers in the Watt level range. This long fiber length gives rise to further nonlinear effects, particularly SBS, that limits the output power in the Raman Stokes wave, due to its hundred times higher gain coefficient [16]. This is due to the backscattered wave removing power from the Raman output, as well as from a narrowband pump.

In this work, suppression of SBS is achieved by using a wideband Raman pump source $(\sim 2.5 \mathrm{THz})$ and by broadening the effective bandwidth of the Raman signal through a WM required for generating the PA signal as well as a simultaneous higher frequency modulation employed outside the acoustic response of the microphone. The use of a wideband pump and signal reduces the Brillouin gain coefficient due to the narrow Brillouin bandwidth of $\sim 50 \mathrm{MHz}$, therefore increasing the overall SBS threshold [22]. The PA sensing modulation frequency and amplitude are determined by the cell geometry and attainment of a modulation index, $m=2$, respectively. In this case, the modulation amplitude is not sufficient to completely suppress SBS, and the modulation frequency does not allow for the total wavelength bandwidth to be contained within the fiber length at any instantaneous point in time. However, the higher frequency suppression modulation allows a number of wavelength bandwidth to be present within the fiber length at any given time, therefore gaining less SBS feedback by reducing the phase matching of scattering events at different positions within the fiber. For a passive fiber the required modulation frequency for the presence of one complete wavelength bandwidth within the fiber can be estimated to be at least $f=c / 2 n L$, with $c$ the speed of light, $n$ the refractive index of the fiber core and $L$ the effective fiber length [22]. However, for the Raman active case used in this work most of the gain and light intensity is distributed at the end of the fiber, which requires a higher frequency to achieve the maximum wavelength distribution over the short fiber length that has the highest SBS influence. A full theoretical analysis to achieve an optimum modulation frequency for this active case is outside the scope of this paper, which focuses on the 


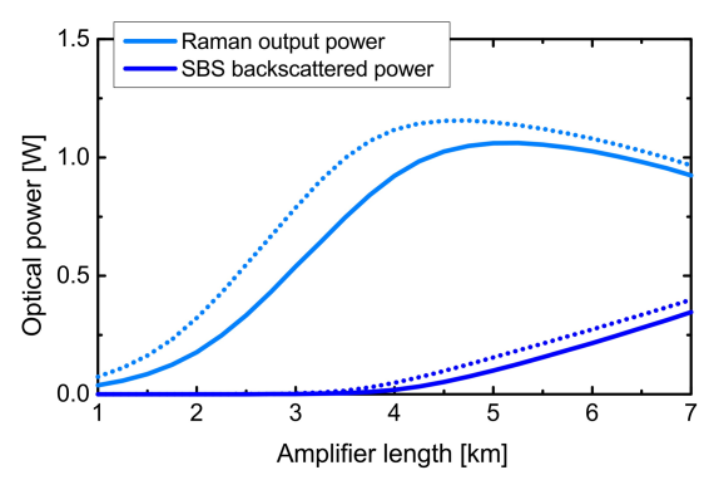

Fig. 2. Simulation of the Raman amplifier signal and SBS backscattered power output for varying active fiber length and $6.5 \mathrm{~mW}$ seed input power (solid lines) or $13 \mathrm{~mW}$ seed input power (dotted lines) using the simulation parameters according to Table I.

application of the Raman amplifier system as source for PA trace gas sensing.

A counter-propagating amplifier design was chosen due to the higher SBS threshold and a lower transfer of intensity noise from the pump to the signal compared to co-propagating designs. To estimate the optimum Raman amplifier fiber length a numerical simulation was constructed using the coupled rate equation system of the Pump, Raman and Brillouin waves [38]:

$$
\begin{aligned}
& \frac{d P_{R}}{d z}=\frac{\varepsilon g_{r} P_{P} P_{R}}{A_{e f f}}-\frac{g_{b, e f f} P_{R} P_{S B S}}{A_{e f f}}-\alpha_{R} P_{R} \\
& \frac{d P_{P}}{d z}=+\frac{\lambda_{R}}{\lambda_{P}} \frac{\varepsilon g_{r} P_{P} P_{R}}{A_{e f f}}+\frac{\lambda_{R}}{\lambda_{P}} \frac{\varepsilon g_{r} P_{P} P_{S B S}}{A_{e f f}}+\alpha_{P} P_{P} \\
& \frac{d P_{S B S}}{d z}=-\frac{\varepsilon g_{r} P_{P} P_{S B S}}{A_{\text {eff }}}-\frac{g_{b, e f f} P_{R} P_{S B S}}{A_{\text {eff }}}+\alpha_{R} P_{S B S} .
\end{aligned}
$$

$P_{R}, \quad P_{P}, \quad P_{S B S}$ are the Raman, Pump and SBS power, respectively, $g_{r}$ is the Raman gain coefficient, $\varepsilon$ is a polarization factor, $g_{b, e f f}$ is the effective Brillouin gain coefficient, $A_{\text {eff }}$ is the effective mode area of the fiber core, $\alpha_{R}$ is the fiber attenuation at the Raman Stokes wavelength $\lambda_{R}, \alpha_{P}$ is the fiber attenuation at the Pump wavelength $\lambda_{P}$ and $z$ is the propagation length in the fiber. The polarization factor for unpolarized propagation, as applied in this paper, is 0.55 . An effective Brillouin gain coefficient was used to account for the SBS suppression through the modulation of the Raman seed laser, considering in this case only the seed laser linewidth broadening through the PA modulation.

The equation system was numerically solved in Matlab for varying Raman amplifier fiber lengths, using a "bvp5c" boundary value solver and the parameters given in Table I. The Raman gain coefficient was obtained by the fiber supplier while the effective Brillouin coefficient was estimated based on the broadening of the laser linewidth by the modulation [16], assuming a laser linewidth corresponding to the WM bandwidth of $\sim 4 \mathrm{GHz}$, which reduces the Brillouin gain estimated from typical fiber parameters as used in [16]. The used boundary conditions are the pump input power at the

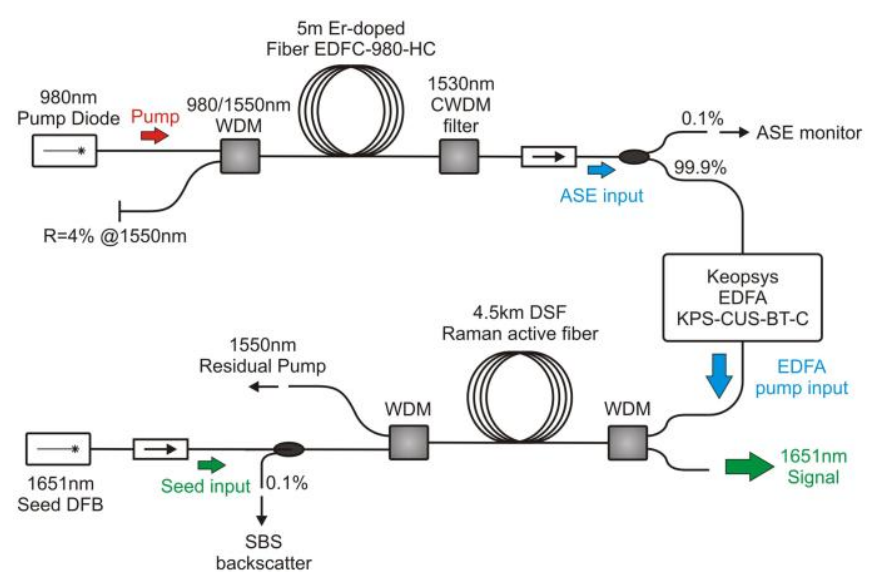

Fig. 3. Schematic of the three-stage Raman amplifier system, using a $5 \mathrm{~mW}$ Er-doped fiber ASE source, a $4 \mathrm{~W}$ commercial pump EDFA and a $4.5 \mathrm{~km}$ long Raman amplifier in a counter-propagation configuration with a $6.5 \mathrm{~mW}$ seed DFB laser at $1651 \mathrm{~nm}$.

amplifier end $P_{P}(L)$, the power of the Raman signal seed at the amplifier start $P_{R}(0)$ and the SBS noise seed power $P_{S B S}(L)$. The use of a Raman signal seed input can reduce the SRS threshold and allows the transfer of modulation characteristics as well as a narrow bandwidth from the seed source to the Raman output. The resulting calculated Raman amplifier output power and backscattered SBS power at the amplifier input are shown in Fig. 2 for varying amplifier lengths. A maximum Raman output power of $1.06 \mathrm{~W}$ can be achieved for a fiber length of $5 \mathrm{~km}$ with a seed laser input power of $6.5 \mathrm{~mW}$. The corresponding SBS backscattered power is $100 \mathrm{~mW}$ at the amplifier input. A threshold behavior for SBS can be seen around $4.5 \mathrm{~km}$ fiber length. Figure 2 also shows the effect of doubling the Raman seed power, which shifts the maximum Raman output power to a shorter amplifier length and simultaneously moves the SBS threshold length to a shorter value. Following the simulation output and the availability of a $6.5 \mathrm{~mW}$ DFB laser as a Raman seed, an amplifier length of $4.5 \mathrm{~km}$ was chosen for the experimental realization of the Raman amplifier system discussed in the next section.

\section{EXPERIMENTAL CHARACTERIZATION OF RAMAN AMPLIFIER SOURCE SYSTEM}

The experimental schematic of the Raman amplifier system is shown in Fig. 3. A $4.5 \mathrm{~km}$ long dispersion shifted fiber (DSF) from Sumitomo was used as the Raman active fiber, with its Raman gain coefficient and attenuation coefficient for the $1530 \mathrm{~nm}$ pump and $1651 \mathrm{~nm}$ signal shown in Table I. The amplifier consists of three pump stages to create the high power pump for the Raman fiber. A $980 \mathrm{~nm}$ single mode pump diode with $20 \mathrm{~mW}$ output power is used to pump an amplified spontaneous emission (ASE) source consisting of a $5 \mathrm{~m}$ long Erbium doped fiber (Nufern EDFC-980-HC). The broad spectrum ASE of the Erbium doped fiber is filtered through a $1530 \mathrm{~nm}$ CWDM filter with a passband of around $20 \mathrm{~nm}$, resulting in an output power of $5 \mathrm{~mW}$ at the second pump stage. The CWDM filter was used to shape the spectrum of the optical signal used as the seed input for a commercial high power EDFA (Keopsys KPS-CUS-BT-C). The 20nm 

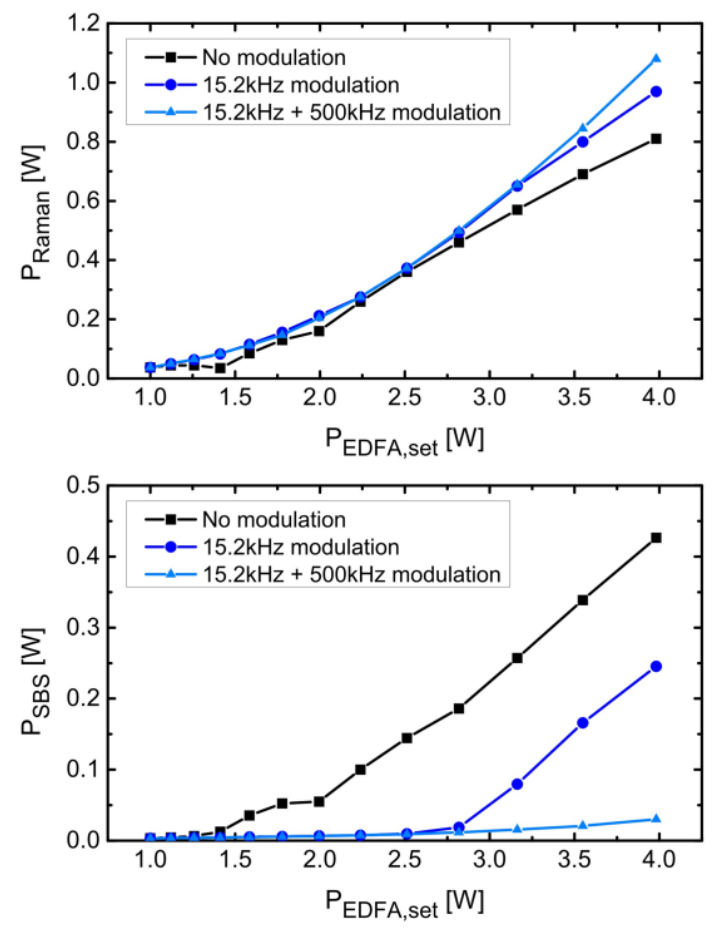

Fig. 4. Comparison of the Raman output signal power $P_{\text {Raman }}$ and back scattered SBS power $P_{S B S}$ of the Raman amplifier system with: using no modulation (squares), using only the photoacoustic resonant modulation (dots), and using the photoacoustic resonant modulation plus a $500 \mathrm{kHz}$ SBS suppression modulation (triangles).

bandwidth was used to suppress SBS originating from the pump inside the Raman amplifier. The high power EDFA has a maximum nominal output power of $4 \mathrm{~W}$, and is used as the counter-propagating pump input for the DSF Raman active fiber through a $1550 \mathrm{~nm} / 1650 \mathrm{~nm}$ WDM. The Raman signal is seeded by a $1651 \mathrm{~nm}$ DFB laser (NEL NLK1U5FAAA) with an output power set point of $6.5 \mathrm{~mW}$. The output of the Raman seed is inserted into the Raman active fiber by a second $1550 \mathrm{~nm} / 1650 \mathrm{~nm}$ WDM at the Raman amplifier input. $0.1 \%$ taps are inserted to monitor the SBS backscatter and the ASE output.

To experimentally characterize the achievable SBS suppression using a frequency modulation in the $100 \mathrm{~s} \mathrm{kHz}$ range, the signal output power, $P_{\text {Raman }}$, and SBS backscatter power, $P_{S B S}$, were measured at the exit and the input of the counter-propagating amplifier respectively. Three operation conditions of the $6.5 \mathrm{~mW}$ seed laser were chosen for comparison; a non-modulated input, an input with a sinusoidal laser drive current modulation at the acoustic resonance of the gas cell, $15.2 \mathrm{kHz}$, and an input with a superimposed sinusoidal modulation of both $15.2 \mathrm{kHz}$ and $500 \mathrm{kHz}$. The modulation amplitude of the $15.2 \mathrm{kHz}$ current dither was controlled in order to maintain a 1f PAS signal with an mvalue of 2 , while the modulation amplitude of the $500 \mathrm{kHz}$ current dither was set to the minimum point where it fully suppressed SBS at the maximum amplifier output power. This minimum amplitude was targeted as high amplitudes reduced the maximum achievable PAS signal amplitude. $P_{\text {Raman }}$ and $P_{S B S}$ for a varying amplifier pump power of the EDFA output $P_{E D F A, s e t}$ are shown in Fig. 4. The Raman threshold is reached

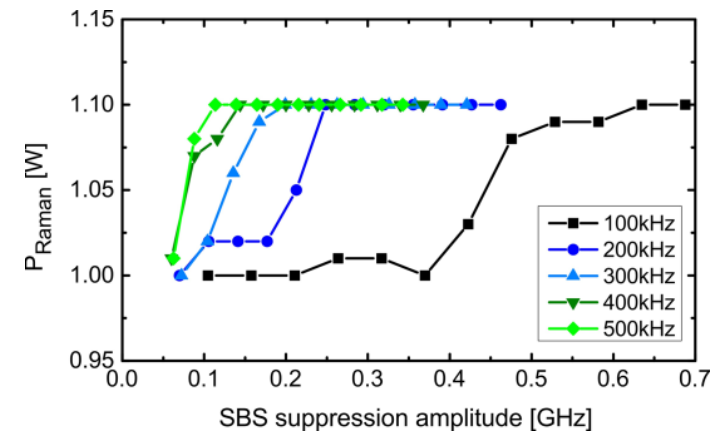

Fig. 5. $1651 \mathrm{~nm}$ Raman output signal power $\mathrm{P}_{\text {Raman }}$ of the Raman amplifier with variation of the SBS suppression modulation frequency and amplitude.

for all operation conditions around $1.5 \mathrm{~W}$ of pump power. The slope efficiency of the amplifier deviates between the operation conditions above approximately $2.5 \mathrm{~W}$ of pump power, with the highest efficiency and highest Raman output power of $1.1 \mathrm{~W}$, for a pump power of $4 \mathrm{~W}$, reached for the double modulation case. The higher slope efficiency is resulting from a better SBS suppression, as shown in the lower graph of Fig. 4. With no applied modulation the SBS threshold is reached almost simultaneously with the Raman threshold at around $1.5 \mathrm{~W}$ of pump power, while a modulation of $15.2 \mathrm{kHz}$ delays the SBS threshold until approximately $2.75 \mathrm{~W}$ of pump power and the combined modulation case does not reach a full SBS threshold, with a maximum SBS backscattered power of $30 \mathrm{~mW}$ at the maximum pump power of $4 \mathrm{~W}$. This two-step increase in the SBS threshold is believed to originate from broadening of the laser linewidth through the $15.2 \mathrm{kHz}$ modulation, which however does not achieve a full simultaneous presence of the broadening in the fiber, plus a higher frequency modulation wavelength spread in the active high power part of the fiber for the $500 \mathrm{kHz}$ modulation as mentioned in section IV.

To further investigate the optimum SBS suppression modulation amplitude and frequency, the Raman output power $P_{\text {Raman }}$ was monitored for modulation frequencies ranging from $100 \mathrm{kHz}$ to $500 \mathrm{kHz}$ and frequency modulation amplitudes $V_{S B S \text { suppression }}$ between $60 \mathrm{MHz}($ at $500 \mathrm{kHz}$ ) and $690 \mathrm{MHz}$ (at $100 \mathrm{kHz}$ ). This SBS suppression modulation gets superimposed with the resonance modulation of $15.2 \mathrm{kHz}$, which has a frequency tuning amplitude of $3.97 \mathrm{GHz}$ to achieve $m=2$. The combined modulation voltage is converted to a drive current modulation to the laser using a bias-T circuit. The resulting $P_{\text {Raman }}$ behavior is shown in Fig. 5. The reduction of SBS and therefore increase in $P_{\text {Raman }}$ can be seen for each modulation frequency. With higher modulation

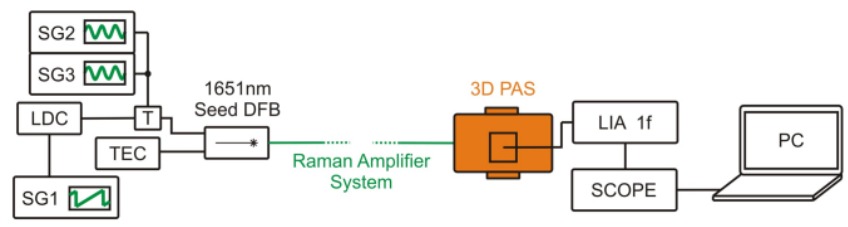

Fig. 6. Schematic of the PAS measurement setup using the Raman amplifier system: LDC, laser diode controller; TEC, temperature controller; SG1, ramp signal generator; SG2\&3, modulation signal generators for the photoacoustic resonant modulation and SBS suppression modulation; T, bias-T; LIA, lockin amplifier; 3D PAS, 3D printed gas cell system. 


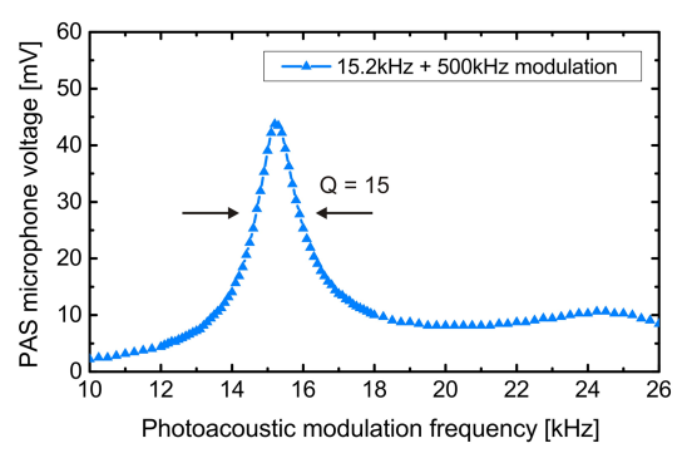

Fig. 7. Measurement of the PAS gas cell resonance using the Raman amplifier source system and $1000 \mathrm{ppm} \mathrm{CH}_{4}$ as sample gas.

frequency a lower $V_{S B S, \text { suppression }}$ amplitude is required to go below the SBS threshold. For $100 \mathrm{kHz}$ SBS is efficiently suppressed around the maximum chosen suppression amplitude of $690 \mathrm{MHz}$, while at $200 \mathrm{kHz}$ an amplitude of around $280 \mathrm{MHz}$ is sufficient, at $300 \mathrm{kHz}$ an amplitude of around $200 \mathrm{MHz}$ and at $400 \mathrm{kHz}$ and $500 \mathrm{kHz}$ an amplitude above around $140 \mathrm{MHz}$. Additionally, with higher modulation frequencies the influence of the SBS suppression modulation on the laser linewidth reduces for a constant modulation amplitude, leading to the choice of a preferred SBS suppression modulation of $500 \mathrm{kHz}$ with an amplitude of $190 \mathrm{MHz}$. The lower required suppression amplitude for higher frequencies is believed to originate again from the potential simultaneous presence of the full wavelength broadening in the high power section of the Raman active fiber, as discussed in section IV, achieving full SBS suppression.

\section{EXPERIMENTAL CHARACTERIZATION OF RAMAN-PAS SYSTEM}

To experimentally characterize the Raman amplifier as a source for the miniaturized 3D printed PAS system (RamanPAS) the experimental setup shown in Fig. 6 is used. The temperature of the $1651 \mathrm{~nm}$ DFB seed laser is controlled through a Thorlabs TED200 and its drive current through a Thorlabs LDC202. A 5Hz ramp signal with $2 \mathrm{Vpp}$ amplitude from signal generator 1 (SG1) is used at the modulation input to create a slow wavelength sweep of $330 \mathrm{pm}$ around the interrogated $\mathrm{CH}_{4}$ gas line at $1650.958 \mathrm{~nm}$. Two sinusoidal modulations at the cell resonance frequency of $15.2 \mathrm{kHz}$ and the SBS modulation frequency, originating from SG2 and SG3 respectively, are added to the laser drive current via a Bias-T circuit. The seed laser output is fed through the Raman amplifier, with the amplifier signal output connected to one of the GRIN lens collimators of the 3D printed gas cell. The microphone signal of the gas cell is demodulated using a Stanford Research Systems SR830 lock-in amplifier with a $1^{\text {st }}$ harmonic demodulation at the cell resonance frequency and then visualized on an Agilent DSO5014A oscilloscope. For all subsequent experimental results the lock-in amplifier time constant was set to $300 \mu$ s and the oscilloscope capture time to $200 \mathrm{~ms}$ with a non-averaged data collection.

To initially characterize the 3D printed gas cell resonance

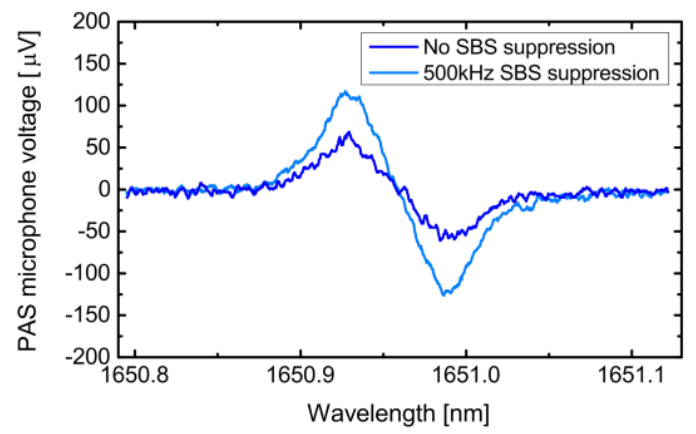

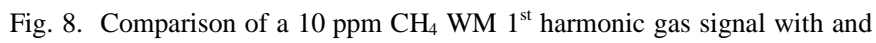
without applying a SBS suppression modulation of $500 \mathrm{kHz}$.

behavior, the cell was filled with a calibrated gas sample of $1000 \mathrm{ppm} \mathrm{CH}_{4}$ using a constant flow rate of $100 \mathrm{ml} / \mathrm{min}$. The EDFA pump power of the Raman amplifier was set to $4 \mathrm{~W}$, with the DFB seed laser set to its bias point of $6.5 \mathrm{~mW}$ at the center of the gas line. The dual modulation consisted of a modulation amplitude of $4 \mathrm{~V}$ at SG2, corresponding to a frequency modulation amplitude of $3.97 \mathrm{GHz}$, combined with a $500 \mathrm{kHz}$ modulation with an amplitude of $1.5 \mathrm{~V}$, corresponding to a frequency modulation amplitude of $190 \mathrm{MHz}$, for the SBS suppression at SG3. The modulation frequency of SG2 was swept from $10 \mathrm{kHz}$ to $26 \mathrm{kHz}$, with the resulting demodulated frequency response of the PAS cell shown in Fig. 7. The first longitudinal resonance of the gas cell can be seen in good agreement with the previously detailed FEM simulation results, with a maximum microphone signal at $15.2 \mathrm{kHz}$ and a resonance Q-factor of 15 . A further small maximum of the frequency response around $25 \mathrm{kHz}$ can be attributed to the nonlinear microphone response including an acoustic Helmholtz resonance created by the microphone air cavities at this point.

With the resonance point of the gas cell confirmed, all further measurements were conducted at cell resonance using a calibrated gas sample of $10 \mathrm{ppm} \mathrm{CH}_{4}$, having an absorbance of $4.3 \cdot 10^{-6} \mathrm{~cm}^{-1}$, again with a constant flow rate of $100 \mathrm{ml} / \mathrm{min}$. To evaluate the influence of the SBS suppression on the recovered gas signal, a direct comparison between applying only the PAS modulation and applying the PAS modulation and the SBS modulation at $500 \mathrm{kHz}$ was performed and is shown in Fig. 8. The only variation between the measurements is a $15.2 \mathrm{kHz}$ modulation with $3.97 \mathrm{GHz}$ amplitude for the non SBS suppressed case and an added SBS suppression modulation of $500 \mathrm{kHz}$ with $190 \mathrm{MHz}$ amplitude for the SBS suppression case. The lock-in amplifier was set to a sensitivity of $500 \mu \mathrm{V}$ and a time constant of $300 \mu$ s with $24 \mathrm{db} /$ oct rolloff. The $1^{\text {st }}$ harmonic gas signal shapes in Fig. 8 show a higher amplitude for the SBS suppressed case, with a signal-to-noise ratio (SNR) of 60 while the SNR of the modulation without SBS suppression is 25 . The increase in SNR can be attributed to both an increase of the Raman signal power from $1 \mathrm{~W}$ to $1.1 \mathrm{~W}$ with suppression of SBS, as shown in Fig. 4, as well as a reduction of the noise content in the Raman output signal, due to the suppression of the noise transfer occurring through SBS [22]. The individual 


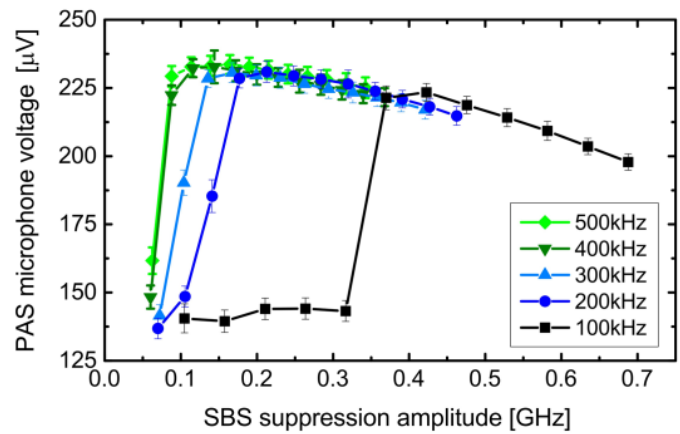

Fig. 9. Microphone signal of the non-averaged Raman-PAS system for a $10 \mathrm{ppm} \mathrm{CH}_{4}$ gas sample with variation of the SBS suppression modulation frequency and amplitude.

contributions to the sensitivity improvement of a factor of 2.4 are a 1f signal amplitude increase by a factor of about 2 and a reduction of the noise floor by about $30 \%$ (see Fig. 8). The signal increase has two origins; the first being the power increase by $10 \%$ and the second originating from SBS transferring optical power into modulation sidebands of the signal which reduces the power present at the PAS modulation frequency. As only this frequency is amplified by the acoustic resonator, and isolated by the lock-in amplifier, this leads to an increase of signal power once SBS is suppressed. The minimum detection limit $(1 \sigma)$ for the case of no applied SBS suppression modulation is then given as $400 \mathrm{ppb} \mathrm{CH}_{4}$, and for the case of using the SBS suppression modulation as $167 \mathrm{ppb}$. The normalized noise equivalent absorption coefficient (NNEA) for this value is calculated as $4.1 \cdot 10^{-9} \mathrm{~cm}^{-1} \mathrm{~W} \mathrm{~Hz}^{-1 / 2}$. It is defined as NNEA $=\alpha_{0} \cdot \mathrm{C}_{\min } \cdot \mathrm{P}_{0} \cdot \Delta \mathrm{f}^{1 / 2}$, using the absorption coefficient $\alpha_{0}=0.358 \mathrm{~cm}^{-1}$ for $\mathrm{CH}_{4}$ at $6057.1 \mathrm{~cm}^{-1}$, the minimum detection limit $\mathrm{C}_{\min }=167 \mathrm{ppb}$, the laser power $\mathrm{P}_{0}=1.1 \mathrm{~W}$ and the lock-in amplifier noise bandwidth $\Delta \mathrm{f}=260 \mathrm{~Hz}$ The small difference between the calculated absorbance at $10 \mathrm{ppm}$ and that inferred from the absorption coefficient quoted above is due to the difference in the broadening coefficients of air and methane used in the calculations..

To confirm and further investigate the optimum SBS suppression condition obtained from the Raman amplifier characterization, the SBS suppression modulation was varied from $100 \mathrm{kHz}$ to $500 \mathrm{kHz}$ over the same steps shown in section V. For each measurement point the SBS suppression modulation amplitude was varied, while the PAS modulation amplitude was kept constant at $3.97 \mathrm{GHz}$. The resulting gas sensor signals were recorded over a timespan of $5 \mathrm{~min}$, with the resulting mean peak-peak value and standard deviation of the signal used for the signal magnitude and error, respectively, shown in Fig. 9. The results are displaying a similar trend as seen for the Raman amplifier output power. The initial microphone signal for low modulation amplitudes and incomplete SBS suppression of around $140 \mu \mathrm{V}$ increased to amplitude values of around $230 \mu \mathrm{V}$ when reaching the SBS suppression condition. Where the Raman amplifier output reaches a stable output power for complete SBS suppression, a trend of slight reducing microphone signal levels can be seen in Fig. 9. Especially for the $100 \mathrm{kHz}$ case a more pronounced

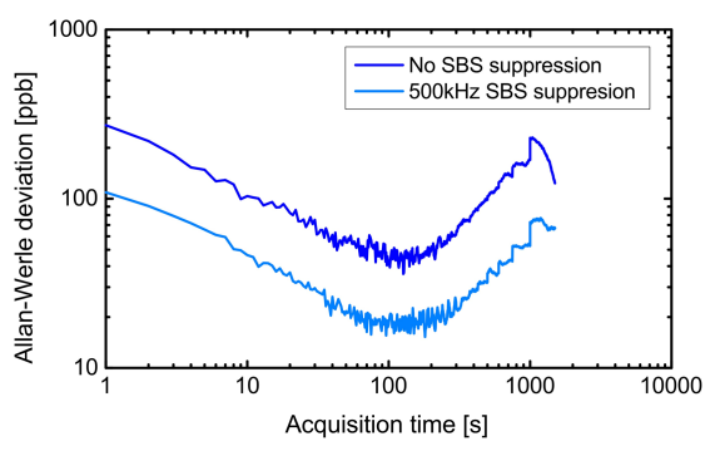

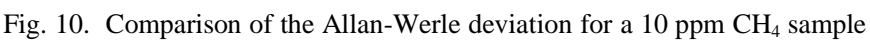
gas concentration with and without applying a SBS suppression modulation of $500 \mathrm{kHz}$ and resulting minimum detection limits $(1 \sigma)$ of $17 \mathrm{ppb}$ and $40 \mathrm{ppb}$, respectively, for $130 \mathrm{~s}$ signal acquisition time.

reduction above a SBS suppression amplitude of $420 \mathrm{MHz}$ is visible, potentially originating from the increased influence of the higher frequency laser linewidth broadening on the PA modulation signal.

So far all detailed measurements were taken without signal averaging and a lock-in amplifier time constant of $300 \mu$ s. To evaluate the overall precision, long term sensor stability and minimum detection limit of the Raman-PAS trace gas sensor an Allan-Werle deviation [39], [40] was calculated as a function of the signal acquisition time. A calibrated sample gas concentration of $10 \mathrm{ppm} \mathrm{CH}_{4}$ was used with a constant flow of $100 \mathrm{ml} / \mathrm{min}$. The gas signals were taken over a time span of 50 min with sampling time intervals of $1 \mathrm{~s}$ and a lockin amplifier sensitivity of $500 \mu \mathrm{V}$. The sampling time intervals are a fivefold of the inverse laser ramp frequency due to the data transfer time of the data acquisition. The resulting AllanWerle plots for both the case of no applied SBS suppression modulation and the case of a SBS suppression modulation with $500 \mathrm{kHz}$ and an amplitude of $190 \mathrm{MHz}$ are shown in Fig.10. In both cases, the detection limit reduces at a similar rate with acquisition times of up to $130 \mathrm{~s}$. After this point the detection limit increases again due to sensor drift. The calculated $1 \sigma$ detection limits with no SBS suppression is reducing from $270 \mathrm{ppb} \mathrm{CH}_{4}$ for an acquisition time of $1 \mathrm{~s}$ to a minimum value of $40 \mathrm{ppb}$ for an acquisition time of $130 \mathrm{~s}$. With the inclusion of the SBS suppression modulation the $1 \sigma$ detection limit is reducing from $110 \mathrm{ppb}$ for an acquisition time of $1 \mathrm{~s}$ to an overall minimum detection limit value of $17 \mathrm{ppb}$ for an acquisition time of $130 \mathrm{~s}$. By employing a SBS suppression modulation the minimum detection limit of the system can therefore be improved by a factor of 2.4. With applying a more representative detection limit of $3 \sigma$, the minimum detectable concentration for the Raman-PAS system was measured as $51 \mathrm{ppb}$ for a signal acquisition time of $130 \mathrm{~s}$. The detection limit for an acquisition time of $1 \mathrm{~s}$ is less than an order of magnitude above the lowest so far reported methane detection limit for PAS, which is $13 \mathrm{ppb}(1 \sigma)$ for an averaging time of $1 \mathrm{~s}$ [4], but uses a near-infrared fiber coupled laser instead of a $7.83 \mu \mathrm{m}$ mid-infrared free space laser. More complex measurement techniques (e.g. cavity enhanced absorption spectroscopy techniques) can reach even lower detection limits, but do not have the advantage of a 
compact 3D printed system with fiber network and multiplex capabilities as shown here.

\section{CONCLUSION}

We have presented the development of a counter-propagating Raman amplifier system at $1651 \mathrm{~nm}$ and its application as a source for miniaturized 3D printed PAS trace gas sensing of $\mathrm{CH}_{4}$. The amplifier design was presented together with a suppression method for the onset of SBS based on a laser modulation in the 100s of $\mathrm{kHz}$ range. The influence of the suppression modulation frequency and amplitude was shown when combined with a $15.2 \mathrm{kHz}$ modulation used for the PAS sensor, leading to a sufficient SBS suppression for a modulation frequency of $500 \mathrm{kHz}$ and amplitude of $190 \mathrm{MHz}$. This source technique for PAS shows the opportunity to be applied for a vast variety of trace gases (e.g. $\mathrm{H}_{2} \mathrm{O}_{2}, \mathrm{CO}, \mathrm{CO}_{2}$, $\mathrm{N}_{2} \mathrm{O}$ ) with the right seed laser and pump wavelength combination. The design and fabrication of the miniaturized 3D printed PAS gas sensor was presented, combined with the experimental characterization of the trace gas sensor when using the Raman amplifier output of $1.1 \mathrm{~W}$ at $1651 \mathrm{~nm}$ as PAS laser source. The influence of the SBS suppression on the gas sensor performance was also evaluated, showing a minimum detection limit $(1 \sigma)$ of $17 \mathrm{ppb}^{\mathrm{CH}_{4}}$ for a sensor acquisition time of $130 \mathrm{~s}$ while using the SBS suppression. These sensor measurement sensitivities are comparable to more complex, bulk optics, techniques such as Cavity Ringdown Spectroscopy or Herriott Cells [41]. The SBS suppression was shown to increase the sensor performance by a factor of 2.4 and leads to a NNEA of $4 \cdot 1 \cdot 10^{-9} \mathrm{~cm}^{-1} \mathrm{~W} \mathrm{~Hz}^{-1 / 2}$. The use of a fiber based source system and delivery, combined with a miniaturized sensor, will allow the implementation in a remote sensing configuration with the potential to multiplex multiple miniature sensors into a full distributed network.

\section{ACKNOWLEDGMENT}

The authors would like to thank Dr. E. Boyd from Wolfson Microelectronics, Edinburgh, UK for donating the flexi-PCB mounted MEMS microphones.

\section{REFERENCES}

[1] W. Ren, W. Jiang, N. P. Sanchez, P. Patimisco, V. Spagnolo, C. Zah, F. Xie, L. C. Hughes, R. J. Griffin, and F. K. Tittel, "Hydrogen peroxide detection with quartz-enhanced photoacoustic spectroscopy using a distributed-feedback quantum cascade laser," Appl. Phys. Lett., vol. 104, no. 4, p. 041117, Jan. 2014.

[2] T. Schmid, "Photoacoustic spectroscopy for process analysis.," Anal. Bioanal. Chem., vol. 384, no. 5, pp. 1071-1086, Mar. 2006.

[3] A. L. Ulasevich, A. V. Gorelik, A. A. Kouzmouk, and V. S. Starovoitov, "A compact resonant $\Pi$-shaped photoacoustic cell with low window background for gas sensing," Appl. Phys. B, vol. 117, no. 2, pp. 549-561, Jun. 2014.

[4] M. Jahjah, W. Jiang, N. P. Sanchez, W. Ren, P. Patimisco, V. Spagnolo, S. C. Herndon, R. J. Griffin, and F. K. Tittel, "Atmospheric CH_4 and N_2O measurements near Greater Houston area landfills using a QCLbased QEPAS sensor system during DISCOVER-AQ 2013," Opt. Lett., vol. 39, no. 4, pp. 957-960, Feb. 2014.

[5] A. G. Bell, "On the production and reproduction of sound by light," Am. J. Sci., vol. s3-20, no. 118, pp. 305-324, Oct. 1880.

[6] V. Spagnolo, P. Patimisco, S. Borri, G. Scamarcio, B. E. Bernacki, and J. Kriesel, "Part-per-trillion level SF6 detection using a quartz enhanced photoacoustic spectroscopy-based sensor with single-mode fiber-coupled quantum cascade laser excitation.," Opt. Lett., vol. 37, no. 21, pp. 44614463, Nov. 2012.

[7] S. L. Firebaugh, K. F. Jensen, and M. A. Schmidt, "Miniaturization and integration of photoacoustic detection," J. Appl. Phys., vol. 92, no. 3, pp. 1555-1563, 2002.

[8] R. Bauer, G. Stewart, W. Johnstone, E. Boyd, and M. Lengden, "3Dprinted miniature gas cell for photoacoustic spectroscopy of trace gases," Opt. Lett., vol. 39, no. 16, pp. 4796-4799, Aug. 2014.

[9] C. B. Hirschmann, J. Lehtinen, J. Uotila, S. Ojala, and R. L. Keiski, "Subppb detection of formaldehyde with cantilever enhanced photoacoustic spectroscopy using quantum cascade laser source," Appl. Phys. B, vol. 111, no. 4, pp. 603-610, Mar. 2013.

[10]P. Patimisco, G. Scamarcio, F. K. Tittel, and V. Spagnolo, "Quartzenhanced photoacoustic spectroscopy: a review.," Sensors (Basel)., vol. 14, no. 4, pp. 6165-6206, Jan. 2014.

[11] L. Dong, H. Wu, H. Zheng, Y. Liu, X. Liu, W. Jiang, L. Zhang, W. Ma, W. Ren, W. Yin, S. Jia, and F. K. Tittel, "Double acoustic microresonator quartz-enhanced photoacoustic spectroscopy," Opt. Lett., vol. 39, no. 8, pp. 2479-2482, Apr. 2014.

[12] R. J. Mears, L. Reekie, I. M. Jauncey, and D. N. Payne, "Low-noise erbium-doped fibre amplifier operating at $1.54 \mu \mathrm{m}$," Electron. Lett., vol. 23, no. 19 , pp. 1026-1028, 1987.

[13]R. Paschotta, J. Nilsson, a. C. Tropper, and D. C. Hanna, "Ytterbiumdoped fiber amplifiers," IEEE J. Quantum Electron., vol. 33, no. 7, pp. 1049-1056, Jul. 1997.

[14]M. E. Webber, M. Pushkarsky, and C. K. N. Patel, "Fiber-amplifierenhanced photoacoustic spectroscopy with near-infrared tunable diode lasers.," Appl. Opt., vol. 42, no. 12, pp. 2119-26, Apr. 2003.

[15] J. P. Besson, S. Schilt, E. Rochat, and L. Thévenaz, “Ammonia trace measurements at ppb level based on near-IR photoacoustic spectroscopy," Appl. Phys. B Lasers Opt., vol. 85, no. 2-3, pp. 323-328, 2006.

[16] G. P. Agrawal, Nonlinear Fiber Optics, 4th ed. Elsevier, 2006.

[17]R. H. Stolen, "The Early Years of Fiber Nonlinear Optics," J. Light. Technol., vol. 26, no. 9, pp. 1021-1031, May 2008.

[18] L. Zhang, H. Jiang, S. Cui, J. Hu, and Y. Feng, "Versatile Raman fiber laser for sodium laser guide star," Laser Photon. Rev., vol. 8, no. 6, pp. 889-895, Nov. 2014.

[19] M. N. Islam, "Raman amplifiers for telecommunications," IEEE J. Sel. Top. Quantum Electron., vol. 8, no. 3, pp. 548-559, May 2002.

[20] J. A. Nagel, V. Temyanko, J. Dobler, E. M. Dianov, A. S. Biriukov, A. A. Sysoliatin, R. A. Norwood, and N. Peyghambarian, "High-Power Narrow-Linewidth Continuous-Wave Raman Amplifier at 1.27 um," IEEE Photonics Technol. Lett., vol. 23, no. 9, pp. 585-587, May 2011.

[21]R. Engelbrecht, "Analysis of SBS Gain Shaping and Threshold Increase by Arbitrary Strain Distributions," J. Light. Technol., vol. 32, no. 9, pp. 1689-1700, May 2014.

[22]D. A. Fishman and J. A. Nagel, "Degradations due to stimulated Brillouin scattering in multigigabit intensity-modulated fiber-optic systems," $J$. Light. Technol., vol. 11, no. 11, pp. 1721-1728, 1993.

[23]R. Arndt, "Analytical line shapes for Lorentzian signals broadened by modulation," J. Appl. Phys., vol. 36, no. 8, pp. 2522-2524, 1965.

[24] J. Reid and D. Labrie, "Second-harmonic detection with tunable diode lasers - Comparison of experiment and theory," Appl. Phys. B, vol. 26, no. 3, pp. 203-210, 1981.

[25] J. A. Silver, "Frequency-modulation spectroscopy for trace species detection: theory and comparison among experimental methods.," Appl. Opt., vol. 31, no. 6, pp. 707-717, 1992.

[26] J. M. Supplee, E. A. Whittaker, and W. Lenth, "Theoretical description of frequency modulation and wavelength modulation spectroscopy.," Appl. Opt., vol. 33, no. 27, pp. 6294-6302, 1994.

[27] P. Kluczynski and O. Axner, "Theoretical description based on Fourier analysis of wavelength-modulation spectrometry in terms of analytical and background signals.," Appl. Opt., vol. 38, no. 27, pp. 5803-5815, 1999.

[28] S. Schilt, L. Thevenaz, and P. Robert, "Wavelength Modulation Spectroscopy: Combined Frequency and Intensity Laser Modulation," Appl. Opt., vol. 42, no. 33, pp. 6728-6738, 2003.

[29] G. Stewart, W. Johnstone, J. Bain, K. Ruxton, and K. Duffin, "Recovery of Absolute Gas Absorption Line Shapes Using Tuneable Diode Laser Spectroscopy with Wavelength Modulation - Part I: Theoretical Analysis," J. Light. Technol., vol. 29, no. 6, pp. 811-821, 2011.

[30] J. R. P. Bain, W. Johnstone, K. Ruxton, G. Stewart, M. Lengden, and K. Duffin, "Recovery of Absolute Gas Absorption Line Shapes Using Tunable Diode Laser Spectroscopy With Wavelength Modulation-Part 


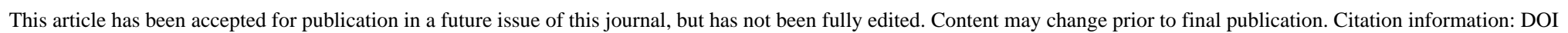
10.1109/JLT.2015.2443377, Journal of Lightwave Technology

JLT-17489-2015

2: Experimental Investigation," J. Light. Technol., vol. 29, no. 7, pp. 987996, Apr. 2011.

[31] M. Wolff and H. Harde, "Photoacoustic spectrometer based on a DFBdiode laser," Infrared Phys. Technol., vol. 41, no. 5, pp. 283-286, 2000.

[32] J.-P. Besson, S. Schilt, and L. Thévenaz, "Multi-gas sensing based on photoacoustic spectroscopy using tunable laser diodes.," Spectrochim. Acta. A. Mol. Biomol. Spectrosc., vol. 60, no. 14, pp. 3449-3456, 2004

[33] S. Schilt and L. Thévenaz, "Wavelength modulation photoacoustic spectroscopy: Theoretical description and experimental results," Infrared Phys. Technol., vol. 48, no. 2, pp. 154-162, Jun. 2006.

[34]M. Angelmahr, A Miklós, and P. Hess, "Wavelength- and amplitudemodulated photoacoustics: comparison of simulated and measured spectra of higher harmonics.," Appl. Opt., vol. 47, no. 15, pp. 2806-2812, 2008.

[35] L. B. Kreuzer, "The physics of signal generation and detection," in Optoacoustic Spectroscopy and Detection, New York: Academic Press, 1977.
[36]F. G. C. Bijnen, J. Reuss, and F. J. M. Harren, "Geometrical optimization of a longitudinal resonant photoacoustic cell for sensitive and fast trace gas detection," Rev. Sci. Instrum., vol. 67, no. 8, pp. 2914-2923, 1996.

[37] H. Wahlquist, "Modulation Broadening of Unsaturated Lorentzian Lines," vol. 1708, no. May 2015, pp. 12-15, 1986.

[38] J. Leng, S. Chen, W. Wu, S. Guo, and X. Xu, "Investigation on the suppression of stimulated Brillouin scattering in single-frequency Raman fiber amplifiers," Opt. - Int. J. Light Electron Opt., vol. 123, no. 8, pp. 659-664, Apr. 2012.

[39]D. W. Allan, "Statistics of atomic frequency standards," Proc. IEEE, vol. 54, no. 2, pp. 221-230, 1966.

[40]P. Werle, "Accuracy and precision of laser spectrometers for trace gas sensing in the presence of optical fringes and atmospheric turbulence," Appl. Phys. B, vol. 102, no. 2, pp. 313-329, Aug. 2010.

[41]J. Hodgkinson and R. P. Tatam, "Optical gas sensing: a review," Meas. Sci. Technol., vol. 24, no. 1, p. 012004, Jan. 2013. 